Philip Manow

\title{
Disproportionalität und ihre Folgen Die Mehrheitswahlkomponente des deutschen Wahlsystems*
}

\section{Kurzfassung}

Der Beitrag untersucht, wie in der Mehrheitswahlkomponente des deutschen Wahlsystems (Erst-)Stimmen in (Direkt-)Mandate übersetzt werden. Während sich generell das bekannte Muster einer disproportionalen, der bekannten $\mathrm{Ku}$ busregel folgenden Übersetzung von Stimmen in Sitze ergibt, zeigt sich doch auch eine Besonderheit, die dem deutschen Mischwahlsystem geschuldet ist: Da die Zahl der Wahlkreisparteien nicht gleich zwei ist, da also Duvergers Gesetz blockiert ist, reichen Erststimmenanteile von deutlich unter $50 \%$, um eine hohe Anzahl von Wahlkreisen zu gewinnen. Im Mittelpunkt des Aufsatzes stehen die Folgen der Disproportionalität, insbesondere die Auswirkungen auf die interne Zusammensetzung der großen Regierungs- und Oppositionsfraktion nach Listen- und Direktkandidaten, auf die Nominierungsstrategien der Parteien und auf die Wahrscheinlichkeit des Entstehens von Überhangmandaten.

* Eine frühere Version dieses Aufsatzes wurde im Political Economy Seminar der Universität Essex vorgestellt. Ich danke allen Diskussionsteilnehmern, insbesondere Vera Troeger und Thomas Plümper, für hilfreiche Kommentare. Ich bin ebenfalls zwei anonymen Gutachten der Zeitschrift für Politikwissenschaft für ihre hilfreichen Gutachten dankbar. Dank auch an Nick Borgwardt für Hilfe bei der Kubusregel-Funktion. 


\section{Inhalt}

1. Fragestellung

2. Die Disproportionalität in der Mehrheitswahlkomponente des deutschen Wahlsystems

a) Der „kritische Mehrheitswert“ $\beta$

b) Die Steigung der Cube-Law Funktion $-\rho$

c) Ein parteipolitischer Bias? $\rho$ und $\beta$ im Vergleich zwischen den Parteien

3. Folgen der Disproportionalität

4. Zusammenfassung

\section{Fragestellung}

24 Überhangmandate in der Wahl zum 17. Deutschen Bundestag - noch nie hatte eine Bundestagswahl eine so hohe Zahl dieser Zusatzmandate zur Folge. Diese vorauszusehende und auch recht präzise prognostizierte hohe Zahl an Überhangmandaten ${ }^{1}$ hatte schon vor der Wahl die politische (und akademische) Diskussion befeuert, insbesondere da lange Zeit denkbar erschien, dass Überhangmandate im neuen Bundestag über die Regierungsmehrheit entscheiden könnten. Die vom Bundesverfassungsgericht angemahnte, bis $2011 \mathrm{zu}$ erfolgende Wahlrechtsreform, bei der insbesondere das mit den Überhangmandaten im Zusammenhang stehende Problem des negativen Stimmengewichts vom Gesetzgeber zu beheben ist, sollte - so lautete die Forderung insbesondere der Grünen und der SPD - vorgezogen und damit bereits die Wahl zum 17. Bundestag von einem kontroversen Element im deutschen Wahlrecht, vom Verdacht einer „manufactured majority“, befreit werden. Die Diskussion über Überhangmandate wurde jedoch nicht erst im Wahlkampf der vergangenen Bundestagswahl wiederbelebt. Seitdem - insbesondere nach der Wiedervereinigung - die Zahl dieser Zusatzmandate sprunghaft angestiegen ist (1990: 6, 1994: 16, 1998: 13, 2002: 5, 2005: 16; 2009: 24), stehen sie erneut im Mittelpunkt des akademischen - politikwissenschaftlichen wie juristischen - Interesses. $^{2}$

1 Behnkes kurz vor der Wahl veröffentlichte Simulationsrechnungen für die 2009er-Bundestagswahl prognostizierten im Mittel 23 Überhangmandate (Behnke 2009).

2 Naundorf 1996; Mager/Uerpmann 1995; Behnke 2003a, 2003b, 2003c, 2009; Grotz 2000; Fürnberg/Knothe 2009; Jesse 1998; Bücking 1998; Mann 1995. Noch im Vereinigungsjahr 1990 konnte Eckhard Jesse schreiben: „Overhang seats [...] in practice [...] are very rare“ (1990: 72). 
Im deutschen gemischten Wahlsystem ${ }^{3}$ werden Überhangmandate c. p. umso wahrscheinlicher, je disproportionaler die Übersetzung von Erststimmen in Direktmandate ist. Gibt es eine starke Drittpartei, kann eine Partei auch mit einem relativ niedrigen Erststimmenanteil eine große Zahl von Wahlkreisen in einem Land gewinnen (Behnke 2003a, 2003b). Diese Bedingung ist insbesondere seit der Wiedervereinigung in vielen Bundesländern erfüllt (Grotz 2000). Damit steigt auch die Wahrscheinlichkeit, dass eine Partei weit mehr Direktmandate erringt, als ihr nach ihrem Zweitstimmenanteil zustehen würde: Überhangmandate entstehen. Aber wie disproportional übersetzen sich in der Mehrheitswahlkomponente des deutschen Mischwahlsystems Stimmen (Erststimmen) in Sitze (Direktmandate) und welche Faktoren bestimmen das Ausmaß der von Wahl zu Wahl variierenden Disproportionalität? Dieser Frage geht dieser Aufsatz in seinem ersten Teil nach. Einer der Faktoren, der dabei im Mittelpunkt steht, ist die „effektive Zahl der Wahlkreisparteien“, also die Zahl derjenigen Parteien, die sich in einem Wahlkreis um das Direktmandat bewerben. Das Ausmaß der Abweichung von einer proportionalen Übersetzung von Stimmanteilen in Sitzanteile ist offensichtlich u. a. abhängig von der Zahl der Parteien, die um Erststimmen konkurrieren - je höher die Zahl der Wahlkreisparteien, desto höher kann die Disproportionalität ausfallen.

Eine nähere Betrachtung der Disproportionalität im Mehrheitswahlelement des deutschen Wahlsystems ist aber nicht nur durch die aktuelle Debatte um Überhangmandate veranlasst. Auch jenseits der Frage, ob Überhangmandate entstehen oder nicht, ist die Disproportionalität in der Mehrheitswahl von Interesse. Sie ist etwa von Bedeutung - wie im Folgenden gezeigt wird - für die interne Zusammensetzung der größten Regierungs- und der größten Oppositionsfraktion. Übersetzt sich der Zugewinn an Erststimmen in einen überproportionalen Zugewinn an Direktmandaten (und umgekehrt: übersetzt sich der Erststimmenverlust in einen überproportionalen Verlust an Direktmandaten), so spiegelt sich das in der internen Zusammensetzung der Parlamentsfraktionen wider. Die jeweils in einer Bundestagswahl erfolgreichere der beiden Volksparteien CDU/CSU und SPD ist überproportional erfolgreich im Gewinnen von Direktmandaten. Sie ist aber auch im Regelfall diejenige Partei, die an der

3 Zur Terminologie: wenn im Folgenden von „Mischwahlsystem“ oder „gemischtem Wahlsystem" die Rede ist, ist damit keine typisierende Einordnung des deutschen Wahlsystems beabsichtigt, auch keine, die sich von der Bezeichnung „System der personalisierten Verhältniswahl“ abgrenzt. Im Kontext der Fragestellung dieses Aufsatzes genügt es festzustellen, dass das deutsche Wahlsystem eine Verhältniswahl- und eine Mehrheitswahlkomponente hat, und dass beide sich wechselseitig beeinflussen - mit Folgen für Kandidaten, Parteien und Wähler. 
nächsten Regierung beteiligt sein wird. Dadurch setzt sich die große Regierungsfraktion im Bundestag mehrheitlich aus Direktkandidaten, die große Oppositionsfraktion hingegen überwiegend aus Listenkandidaten zusammen. Dies hat $u$. a. Folgen für die Nominierungsstrategien der Parteien, die nicht nur bei Stimmenverluste ihre Direktkandidaten über sichere Listenplätze absichern müssen, sondern auch bei Stimmengewinnen ihre Listenkandidaten vor dem Erfolg der eigenen Direktkandidaten „schützen“ müssen. Diese Folgen der Disproportionalität der Mehrheitswahlkomponente im deutschen Mischwahlsystem werden im zweiten Teil dieses Aufsatzes näher beleuchtet.

Der Beitrag ist wie folgt gegliedert: Ich beschreibe zunächst empirisch, wie bei Bundestagswahlen Erststimmen in Direktmandate übersetzt werden. Die Beschreibung ist angeleitet durch folgende Fragen: Hat das Ausmaß der Disproportionalität in der Mehrheitswahlkomponente des deutschen Wahlsystems im Verlauf der Zeit zugenommen? Wie hat sich der durchschnittliche Erststimmenanteil, der zum Erringen eines Direktmandats ausreicht, verändert? Treten ,polarisierte“ Erststimmenresultate, bei denen eine Partei alle, die andere keine der möglichen Direktmandate in einem Land erringt, im Zeitverlauf häufiger auf? Bringt die Disproportionalität parteipolitische Verzerrungseffekte mit sich, d. h. benachteiligt oder bevorzugt sie CDU/CSU oder SPD?

Mein Untersuchungszeitraum umfasst von 1953 bis 2009 alle 16 Bundestagswahlen, in denen der Wähler mit der Erststimme Direktkandidaten im Wahlkreis und mit der Zweitstimme Parteilandeslisten wählen konnte. Die erste Bundestagswahl wird wegen ihres abweichenden Wahlrechts nicht weiter berücksichtigt. Ich betrachte zudem ausschließlich die zwei Parteien, die in der weit überwiegenden Mehrzahl der Fälle in der Lage gewesen sind, ein Direktmandat zu erringen, die SPD und die CDU/CSU. Da der Abgleich zwischen Zweit- und Erststimmen auf der Landesebene stattfindet, liegen den folgenden Auswertungen 390 Beobachtungen zugrunde: 16 Wahlen werden insgesamt betrachtet (alle Bundestagswahlen ohne die 1949er-Wahl), dabei eine Wahl mit 9 Bundesländern (1953) ${ }^{4}$, neun Wahlen mit 10 Bundesländern (1957-1987), und sechs Wahlen mit 16 Bundesländern (seit 1990). Erststimmen und Direktmandate werden jeweils für die Union und die SPD ausgewiesen, sodass der komplette Datensatz $2 \times(9+9 \times 10+6 \times 16)=390$ Beobachtungen umfasst.

Im zweiten Teil des Aufsatzes geht es dann um die Folgen der Disproportionalität: Wie wirkt sie sich auf die Zusammensetzung der jeweils großen Regie-

4 Ohne das Saarland.

5 Die Berliner Abgeordneten wurden vom Berliner Abgeordnetenhaus entsprechend der Fraktionsstärke in den Bundestag entsandt. 
rungsfraktion und der größten Oppositionsfraktion aus, wie auf die Nominierungsstrategien der Parteien? Wie beeinflusst sie die Sicherheit von Listen- und Wahlkreiskandidaturen? Wie bestimmt sie das Auftreten von Überhangmandaten? Eine kurze Zusammenfassung schließt den Aufsatz ab.

\section{Die Disproportionalität in der Mehrheitswahlkomponente des deutschen Wahlsystems}

Die Disproportionalität der Mehrheitswahl folgt bekanntlich häufig dem Muster eines Polynoms dritter Ordnung. Dieser Sachverhalt wird in der Politikwissenschaft unter dem Begriff des Cube Law oder der Cube Rule diskutiert (Taagepera/Shugart 1989). In seiner einfachsten Form postuliert es den folgenden Zusammenhang zwischen dem Stimmenanteil v einer Partei i und ihrem Sitzanteil s:

$$
\left(\frac{s_{i}}{1-s_{i}}\right)=\left(\frac{v_{i}}{1-v_{i}}\right)^{3}
$$

Der Exponentialkoeffizient, der die Steigung der Funktion bestimmt, ist dabei grundsätzlich nur empirisch zu bestimmen, sodass in ihrer allgemeinen Form sich die Cube Rule-Formel wie folgt darstellt:

$$
\left(\frac{s_{i}}{1-s_{i}}\right)=\left(\frac{v_{i}}{1-v_{i}}\right)^{\rho}
$$

Dass das Verhältnis der Parlamentssitze zweier Parteien in etwa dem Verhältnis ihrer Stimmenanteile in dritter Potenz entspricht, wurde erstmals für das britische Mehrheitswahlrecht beschrieben (Kendall/Stuart 1950, 1952). Empirisch ergeben sich aber häufig Abweichungen von einem Steigungsfaktor 3. So ist selbst für das britische Mehrheitswahlrecht nachgewiesen worden, dass $\rho$ hier im Regelfall näher bei 2,5 als bei 3 liegt (Laakso 1979): „While the cube law is stated deterministically, even a cursory examination of election statistics shows that it does not hold perfectly" (zitiert nach King/Browning 1987: 1258). Die Cube Rule ist daher auch eher als - simple - Orientierungsregel zu 
begreifen, denn als „Gesetz“ (Taagepera/Shugart 1989). Der Parameter $\rho$ bestimmt dabei das Ausmaß „elektoraler Responsivität“ (King 1990) eines Wahlsystems, er bildet seinen ,amplifying effect" (Jesse 1990: 64) ab, d. h. an ihm ist abzulesen, wie überproportional sich ein Stimmenzuwachs in einen $\mathrm{Zu}$ wachs an Sitzen übersetzt. Ist $\rho=1$, handelt es sich um ein perfekt proportionales Wahlsystem, geht $\rho \rightarrow \infty$, handelt es sich um ein ,winner-takes-all“-System. Hier gewinnt die Partei mit der relativen Mehrheit der Stimmen alle Sitze (King 1990; King/Browning 1987).

Betrachten wir das Verhältnis von Erststimmenanteilen und Direktmandateanteilen im deutschen Wahlsystem, so bestätigt sich auf den ersten Blick die Cube Rule in einem erstaunlichen Ausmaß (Abbildung 1; Manow 2010a). Schätzen wir den Steigungsparameter auf der Grundlage von 16 Bundestagswahlen und den Anteilen an Erststimmen und Direktmandaten von SPD und CDU/CSU, so ergibt sich ein $\rho=3,17$ bei einem $R^{2}$ von 0,98 (Manow 2010a: Tabelle 1).

\section{Abbildung 1}

Erststimmen- und Direktmandateanteile für die CDU/CSU und die SPD, 1953-2009 auf Bundesebene

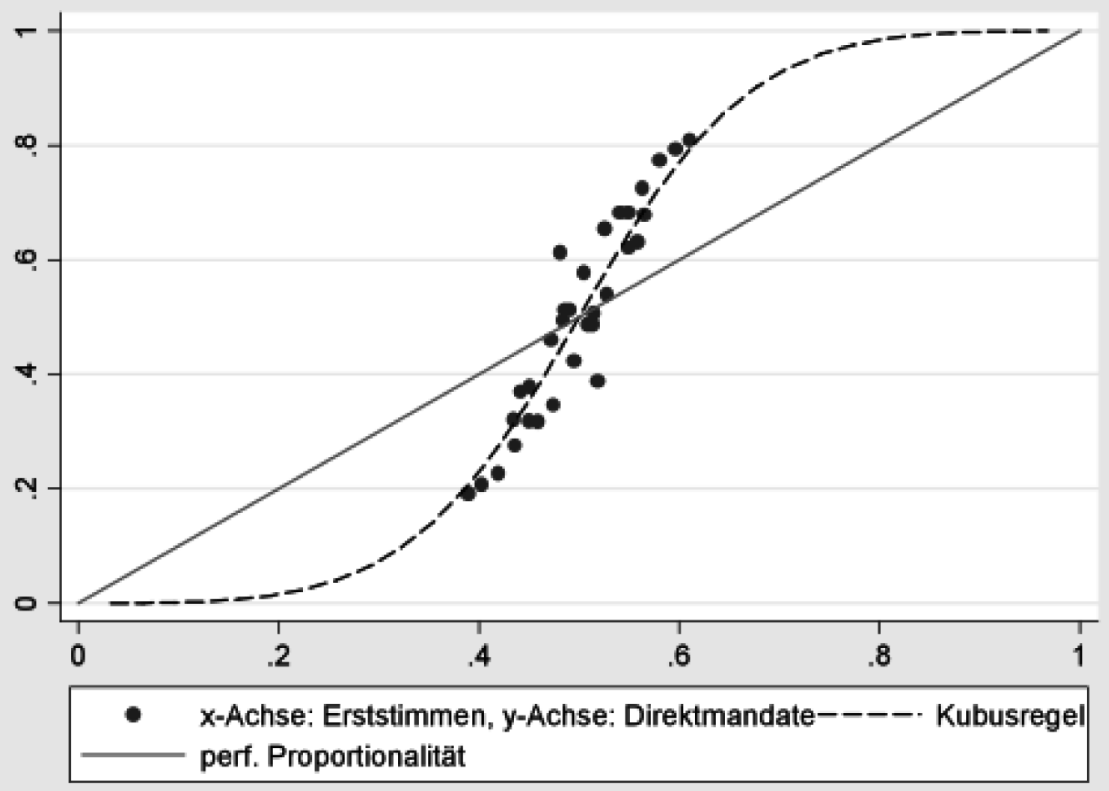


Betrachten wir jedoch den Zusammenhang zwischen Direktmandaten und Erststimmen nicht auf der Bundesebene, sondern auf der für die sogenannte „Unterverteilung" relevanten Landesebene (vgl. § 6 Bundeswahlgesetz), auf der die Verrechnung von Sitzen aufgrund der im Land errungenen Zweitstimmen mit den im Land gewonnenen Direktmandaten vorgenommen wird, dann ergeben sich zwei typische, dem Charakter des deutschen Wahlsystems als einem gemischten Wahlsystem geschuldete Ausprägungen der Kubusregel (Manow 2010a): erstens eine steilere Steigung der Kubusregel-Funktion (also einem größeren $\rho$ ) auf der Landesebene, die insbesondere beeinflusst ist von der Zahl der Wahlkreise pro Bundesland ${ }^{6}$ und zweitens eine bereits bei Erststimmenanteilen von deutlich unter $50 \%$ stark steigende Zahl von gewonnenen Wahlkreisen - weil sich im Wahlkreis in der Regel mehr als zwei Parteien um das Mandat bewerben, also aufgrund des Umstandes, dass Duvergers Gesetz in der Mehrheitswahlkomponente des deutschen Mischwahlsystems nicht oder nur sehr eingeschränkt gilt (Cox/Schoppa 2002).

Damit lässt sich die Kubusregelfunktion um einen weiteren Parameter ergänzen, der diesem Umstand Rechnung trägt. Neben $\rho$, das die ,elektorale Responsivität" der Mehrheitswahl abbildet, also die Steigung der Funktion definiert, nach der sich Stimmen in Sitze übersetzen, ist ein weiterer Parameter nötig, der die Abweichungen von einer strikt proportionalen Übersetzung bestimmt. Dieser Parameter erfasst die Linksverschiebung der Funktion, an der sich ersehen lässt, wie sehr eine erfolgreiche Stimmenmehrheit von dem in einem Zwei-Parteien-System vorherrschenden, „klassischen“ $50 \%$-Wert abweicht (kritischer Mehrheitswert; Manow 2010a). Vergleichen wir die auf diese Art erweiterte Kubusregel-Funktion für die beiden großen Parteien, die bislang regelmäßig die weit überwiegende Zahl der Direktmandate errungen haben, also für die SPD und die CDU/CSU, dann können wir ebenfalls ermitteln, ob die Übersetzung von Stimmen in Sitze parteipolitisch neutral oder verzerrt ausfällt (parteipolitische Verzerrung; Bias).

Die zwei Parameter einer modifizierten Kubusregel-Funktion beschreiben also die drei zentralen Dimensionen der Disproportionalität: elektorale Responsivität, kritischer Mehrheitswert und parteipolitische Verzerrung. Die zwei Parameter werden hier mit $\rho$ und $\beta$ bezeichnet. Ihre Rolle bei der Übersetzung von Erststimmen in Direktmandate lässt sich anhand einer erweiterten Kubusregel-Funktion veranschaulichen:

6 Also in der Begrifflichkeit von Taagepera/Shugart von der ,assembly size“ (Taagepera/ Shugart 1989) oder in der Begrifflichkeit von Gary Cox, von der Größe des ,secondary district". 


$$
\left(\frac{s_{i}}{1-s_{i}}\right)=\left(\frac{v_{i}+\beta}{1-v_{i}-\beta}\right)^{\rho}
$$

Methodisch ist wichtig, dass wir die einzelnen Parameter der Cube RuleFunktion nicht isoliert schätzen, wenn wir sie unverzerrt ermitteln wollen. Eine Schätzung von $\rho$ ohne die gleichzeitige Schätzung von $\beta$ würde fehlerhafte Werte ermitteln, wenn es bei der Übersetzung von Stimmen in Sitze zu einer deutlichen Linksverschiebung der Cube Rule-Funktion kommt (King 1990; King/Browning 1987). Ob es dazu kommt, ist jedoch allein empirisch zu bestimmen.

Genau um diese Bestimmung soll es in den folgenden Abschnitten gehen. Dabei lassen sich aus der Literatur folgende Erwartungen formulieren: Für die Mehrheitswahlkomponente im bundesdeutschen Wahlsystem ist zu erwarten, dass die Steigung der Kurve ( $\rho$ ) abhängig ist von der Zahl der Wahlkreise, auf die ein Landeszweitstimmenergebnis umgerechnet werden muss - mit höherer Disproportionalität in Bundesländern mit weniger Wahlkreisen (Taagepera/ Shugart 1989). Der parteipolitische Bias der Mehrheitswahl bestimmt sich - so ist zu erwarten - unter anderem nach der regionalen Zusammensetzung der Wählerschaft. Benachteiligt sind hier Parteien mit vielen Hochburgen, d. h. regional konzentrierter Wählerschaft, weil sie beim Gewinn dieser Wahlkreise relativ viele Stimmen ,verschenken“ (Rodden 2010; Gudgin/Taylor 1979). Ob dies eher zum Nachteil der CDU/CSU oder der SPD ausschlägt, soll nachfolgend empirisch ermittelt werden. Die Linksverschiebung der Cube-Law-Funktion $(\beta)$ ergibt sich insbesondere aus der effektiven Zahl der Wahlkreisparteien, die sich um ein Direktmandat bewerben. Im deutschen Mischwahlsystem beobachten wir in seiner Mehrheitswahlkomponente keine Konvergenz der Parteienzahl auf zwei (Cox/Schoppa 2002) - Duvergers Gesetz ist blockiert, weil auch kleine Parteien im Regelfall mit eigenen Direktkandidaten antreten in der Hoffnung, dies wirke sich positiv auf ihren Zweitstimmenanteil aus (Hainmüller/ Kern/Bechtel 2006; Hainmüller/Kern 2008; Manow 2010b). Mit einer höheren Zahl an Wahlkreisparteien können Direktmandate mit geringerem Erststimmenanteil errungen werden, die Kubusregel-Funktion verschiebt sich nach links: $\beta>0$. Ich werde diese zwei Parameter im Folgenden näher betrachten und für das deutsche Wahlsystem empirisch bestimmen. Ich gehe dabei in umgekehrter Reihenfolge vor und beginne mit der Betrachtung der Linksverschiebung der Kubusregel-Funktion. 


\section{a) Der ,kritische Mehrheitswert“ $\beta$}

Im Folgenden soll zunächst der Effekt der Zahl der Wahlkreisparteien auf den „kritischen Mehrheitswert“ $\beta$ betrachtet werden. Mit dem Anstieg der Zahl der Parteien - so die Hypothese - sollte der Wert, um den herum ein leichter Anstieg der Erststimmen zu einem erheblichen Zugewinn an Direktmandaten und ein leichter Verlust zu einem erheblichen Verlust an Direktmandaten führt, signifikant kleiner als $50 \%$ werden. Den Effekt der Linksverschiebung kann etwa der Vergleich einer „Vor-Vereinigungswahl“ (hier der Bundestagswahl 1983) mit einer Nach-Vereinigungswahl (hier die 2009er-Wahl) gut veranschaulichen (Abbildung 2).

\section{Abbildung 2}

Erststimmenanteile und Anteile an den Direktmandaten von CDU/CSU und SPD auf Landesebene bei den Bundestagswahlen 1983 und 2009

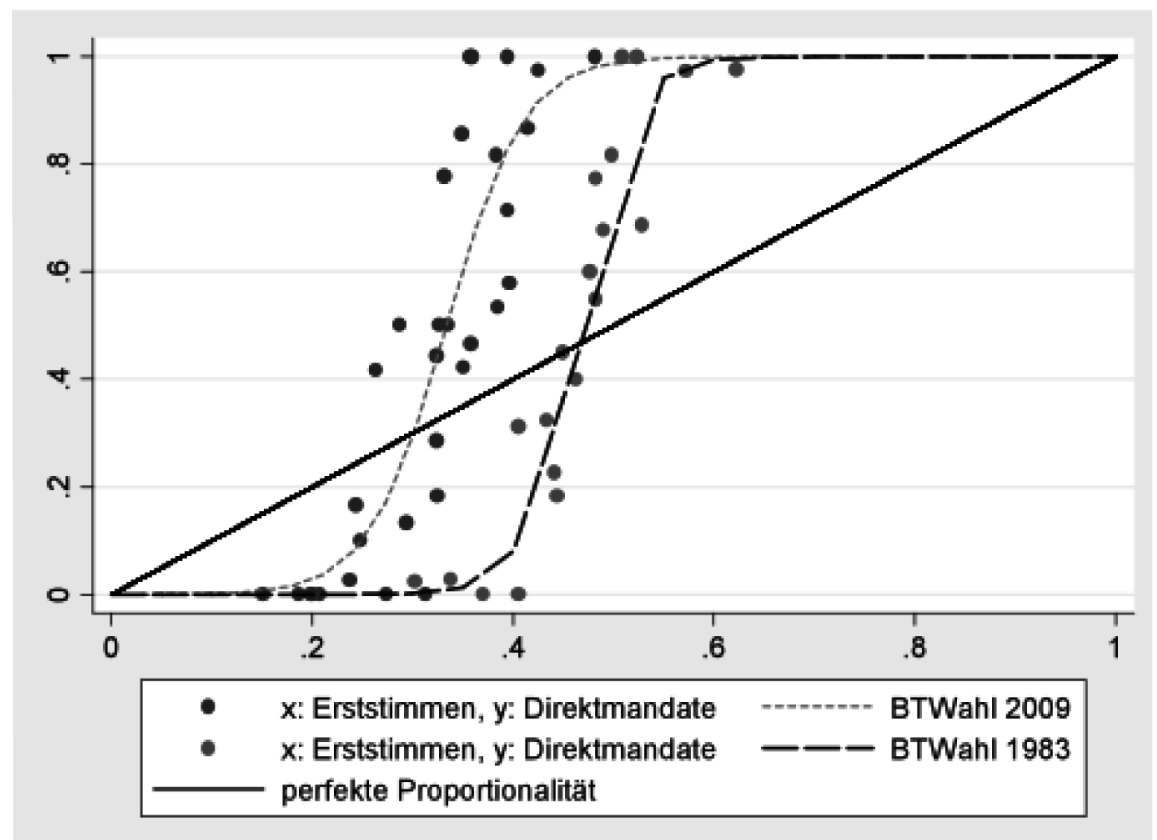

Abbildung 2 zeigt in aller wünschenswerten Deutlichkeit, dass sich der durchschnittliche Erststimmenanteil zur Erlangung einer hohen Zahl von 
Direktmandaten im Zeitverlauf erheblich verringert hat. Schnitt 1983 die Kubusregel-Funktion die Linie perfekter Proportionalität noch knapp unterhalb der $50 \%$ Stimmanteil-Grenze, so liegt der Schnittpunkt 2009 nur noch um die $30 \%$. Die markante Linksverschiebung, die wir hier beim Vergleich der zwei Kurvenverläufe beobachten können, bestätigt sich bei alternativer Betrachtungsweise. Vergleichen wir die Erststimmenanteile, die zum Gewinn des Wahlkreises und damit zum Einzug in den Bundestag ausgereicht haben, so zeigt sich auch hier ein klarer Entwicklungstrend. Abbildung 3 vergleicht die Dichtefunktionen der Erststimmenanteile aller Direktabgeordneten für zwei Vor-Vereinigungsbundestagswahlen (Wahlen zum 8. und 9. Bundestag), mit den Erststimmenanteilen aller Direktabgeordneten zweier Nach-Vereinigungsbundestagswahlen (Wahlen zum 13. und 14. Bundestag).

\section{Abbildung 3}

Dichtefunktionen für die Erststimmenanteile der direkt gewählten Bundestagsabgeordneten, ein Vergleich der 8. und 9. Legislaturperiode (Bundestagswahlen von 1976 und 1980) mit der 13. und 14. Legislaturperiode (Bundestagswahlen von 1994 und 1998)

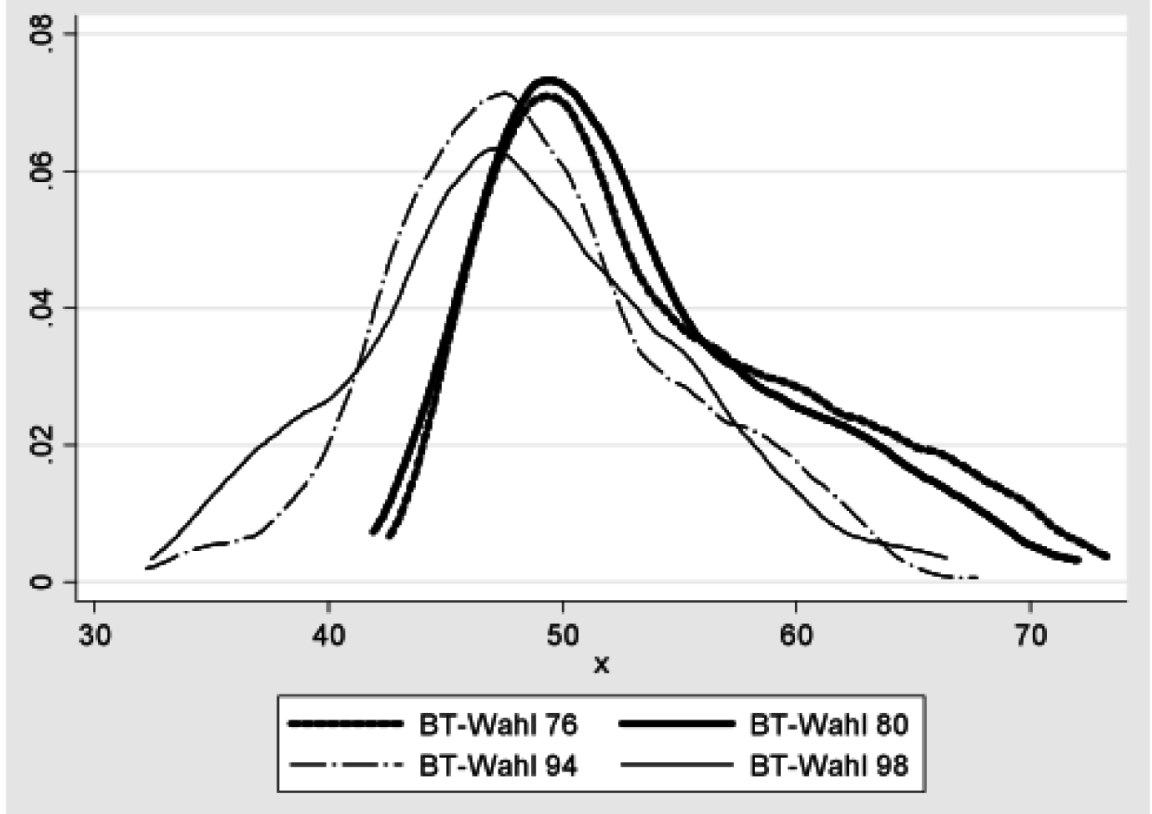


Auch in Abbildung 3 wird eine entsprechende „Linksverschiebung“ für die späteren Bundestagswahlen deutlich sichtbar, d. h. nun gelingt der Einzug in den Bundestag mit einem im Durchschnitt deutlich niedrigeren Erststimmenergebnis. Der Vergleich zeigt insbesondere eine Zunahme der direkt gewählten Abgeordneten mit niedrigem Erststimmenanteil im Bereich von 35 bis $45 \%$. Zugleich nimmt die Streuung des Erststimmenanteils, der zu einem Bundestagsmandat verhilft, zu. Dies führt zusätzlich zu einer flacheren, breiteren Verteilung.

Empirisch zeigt sich die Entwicklung des (gerundeten) $\beta$-Wertes über die 16 Bundestagswahlen hinweg zunächst als Absenkung und dann als erneuter Anstieg: 0,13 (1953), 0,11 (1957), 0,10 (1961), 0,06 (1966), 0,05 (1969), 0,03 (1972), 0,04 (1976), 0,05 (1980), 0,03 (1983), 0,06 (1987), 0,09 (1990), 0,09 (1994), 0,10 (1998), 0,10 (2002), 0,12 (2005), und 0,17 (2009). Diese Zahlen sind direkt zu interpretieren: Ist $\beta=0$, dann liegt die maximale Steigung der Kurve bei $50 \%$ Erststimmenanteil. Ein $\beta$ von größer 0 gibt an, bei welchem Stimmenanteilen von unter $50 \%$, $\rho$ seinen maximalen Wert erreicht. Dabei erweist sich die Zahl der (Wahlkreis-)Parteien als starker und robuster Erklärungsfaktor für die Linksverschiebung der Kurve (Manow 2010a). Erhöht sich die effektive Zahl der Wahlkreisparteien um 1, dann steigt $\beta$ um etwa 0,07 bis 0,08. Dabei lag die durchschnittliche Zahl der Wahlkreisparteien in den ersten Bundestagswahlen bei 3,2, in den 1960er- und 1970er-Jahren bei 2,2, in der letzten Bundestagswahl dann bei 3,7.

Der sprunghafte Anstieg der Überhangmandate nach 1990 ist vor allem ein Hinweis auf die Besonderheiten des Parteiensystems in den ostdeutschen Bundesländern, in denen die Stimmenanteile der beiden großen Volksparteien CDU und SPD aufgrund der Stellung der PDS/Die Linke als starker Drittpartei deutlich unter ihren westdeutschen Vergleichswerten liegen. Dies legt es nahe, für die Bundestagswahlen ab 1990 die Disproportionalität zwischen West und Ost zu vergleichen (Abbildung 4).

Abbildung 4 bestätigt deutlich, dass der Drittparteien-Effekt in den neuen Bundesländern aufgrund der starken Stellung der PDS/Die Linke besonders ausgeprägt ist. Im Vergleich aller Bundestagswahlen seit 1990 fällt der $\beta$-Parameter in den neuen Bundesländern mehr als doppelt so hoch aus wie in den alten Bundesländern $(0,1587 \mathrm{zu} 0,0727)$, während der $\rho$-Parameter sich zwischen West und Ost nicht unterscheidet. 
Abbildung 4

Die Kubusregelfunktion für die Bundestagswahlen 1990-2009, neue und alte Bundesländer im Vergleich

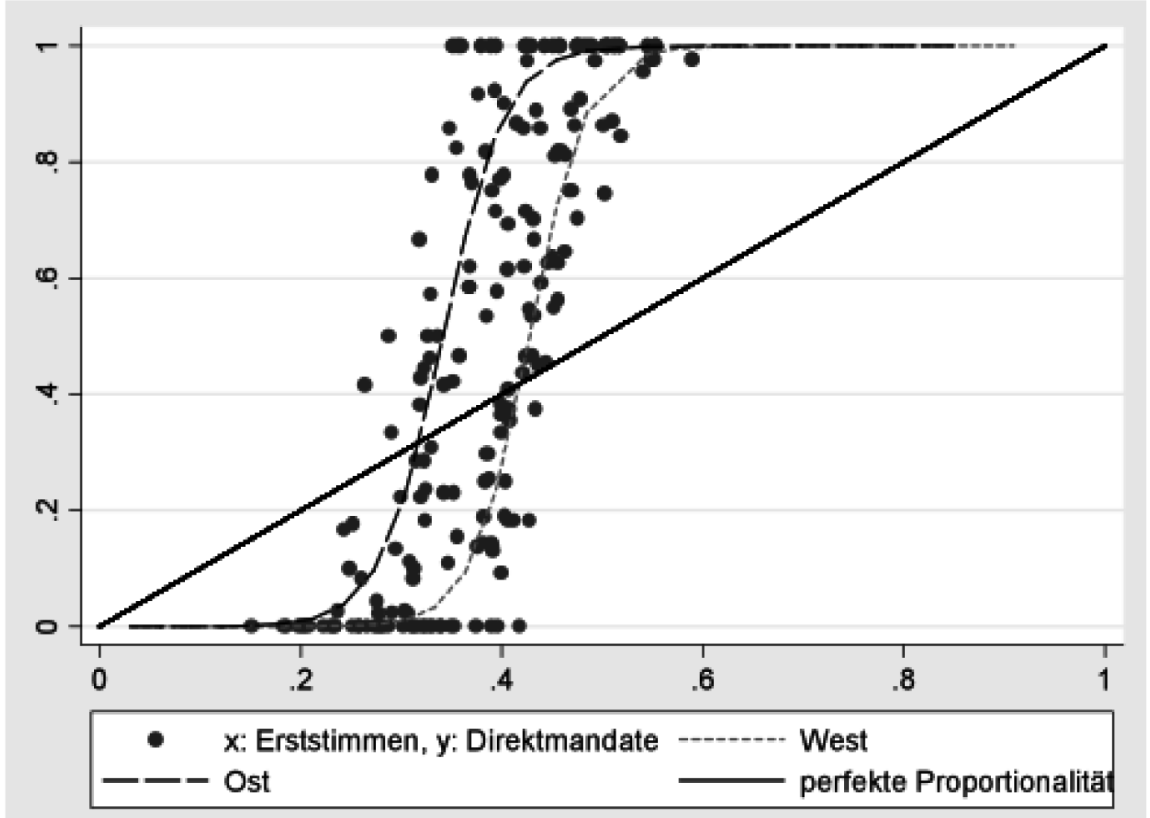

Im folgenden Abschnitt geht es nun um die Schätzung des Steigungsparameters $\rho$.

\section{b) Die Steigung der Cube-Law Funktion - $\rho$}

Betrachtet man den Steigungsparameter für jede der 16 Bundestagswahlen seit 1953, so ergibt sich keine vergleichbar klare Trendentwicklung, wie sie beim $\beta$-Parameter sichtbar wurde (siehe oben, Abschnitt 2 a). Als einzige RegelmäBigkeit zeigt sich in der Betrachtung der einzelnen Bundestagswahlen ein (gerundeter) $\rho$-Wert von - zum Teil deutlich - über $3 .^{7}$ Wenn wir die einzelnen

7 1953: 3,68; 1957: 5,53; 1961: 7,81; 1965: 6,81; 1969: 5,61; 1972: 5,44; 1976: 6,86; 1980: 7,31; 1983: 9,32; 1987: 8,06; 1990: 6,20; 1994: 6,36; 1998: 3,59; 2002: 6,76; 2005: 3,54; 2009: 6,50. 
Bundesländer vergleichen, ergibt sich eine stärkere systematische Varianz dieses Parameters (Manow 2010a). Wie zu erwarten, verläuft die KubusregelFunktion insbesondere in den Ländern mit nur wenigen Wahlkreisen, also insbesondere in den Stadtstaaten Bremen und Hamburg, sehr steil (Taagepera/ Shugart 1989: Kapitel 15). Dies spiegelt den rein arithmetischen Zusammenhang zwischen Proportionalität und „Wahlkreisgröße“ wider - hier die Größe des ,sekundärem Distrikts“, also der Zahl der Wahlkreise pro Bundesland (Cox 1997). Je höher die Zahl der Wahlkreise, desto exakter kann die Sitzverteilung der jeweiligen Stimmverteilung angenähert werden - wobei die maximale Zahl der Sitze immer eine Obergrenze der Proportionalität definiert (Taagepera/ Shugart 1989). Es sollte also einen Unterschied für die Proportionalität der Sitzverteilung machen, ob ein Stimmenergebnis auf 2 Wahlkreise (wie in Bremen 2002 und 2005) oder auf 73 Wahlkreise (wie in Nordrhein-Westfalen in den Bundestagswahlen von 1965 bis 1976) umgerechnet wird. In der Tat lässt sich beim Vergleich der Kubusregelfunktion zwischen den Bundesländern ein systematischer, starker Effekt der Zahl der Wahlkreise auf die Steigung der Kurve feststellen. Mit jedem zusätzlichen Wahlkreis sinkt der Steigungsparameter um 0,32 (bei einer Streuung von $\rho$ zwischen 2,9 in Nordrhein-Westfalen und 55 in Hamburg; vgl. Manow 2010a, Tabellen 2b und 4b).

Dieser Effekt ist erneut in der grafischen Darstellung leicht zu veranschaulichen (Abbildung 5). Vergleichen wir den Stadtstaat Hamburg mit NordrheinWestfalen, so zeigt sich die erhebliche Abweichung des Steigungsparameters (bei ähnlichem $\beta: 8,6[\mathrm{HH}]$ und 5,8 [NRW]). Sie wird vor allem verursacht durch die Zahl der Wahlkreise, auf die das Erst- und das Zweitstimmenergebnis umgerechnet werden müssen. 
Abbildung 5

Die Kubusregel-Funktion für Hamburg und Nordrhein-Westfalen, 16 Bundestagswahlen 1953-2009

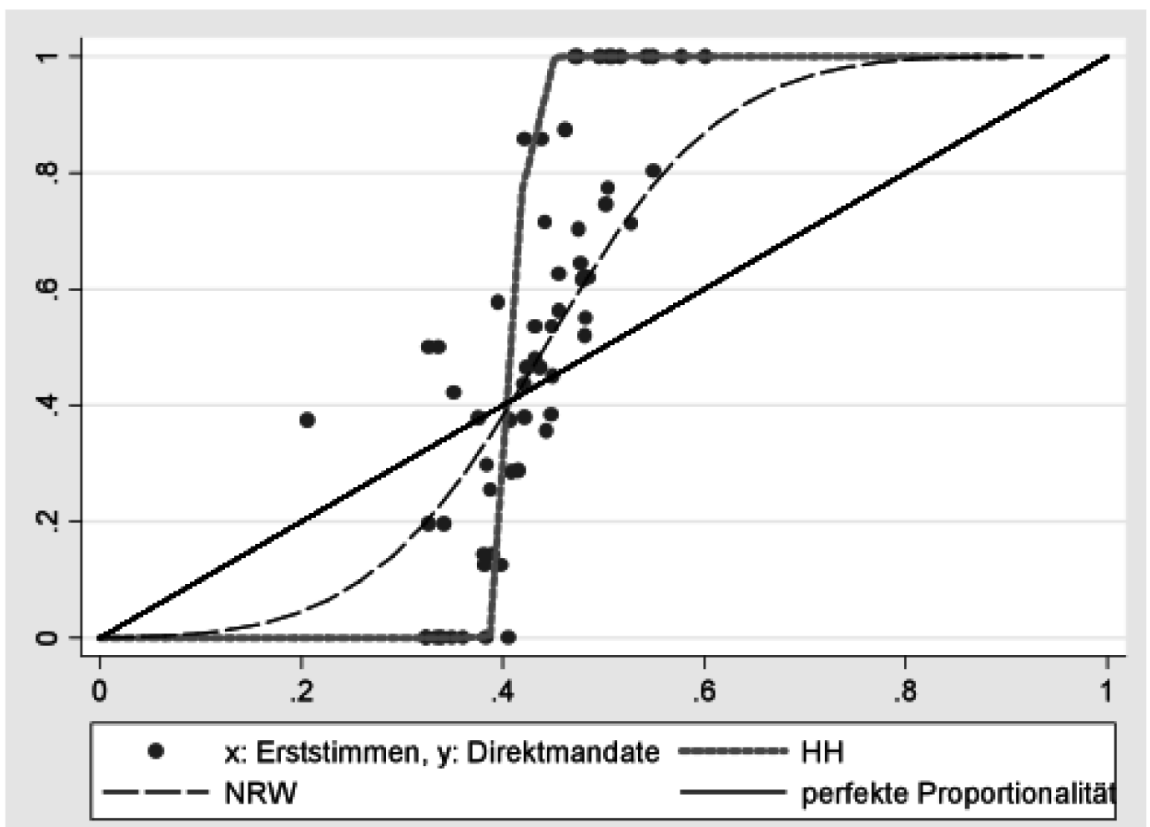

Im nachfolgenden Abschnitt geht es um die Frage, ob die Übersetzung von Stimmen in Sitze in der Mehrheitskomponente des deutschen Mischwahlsystems parteipolitisch neutral oder aber verzerrt ist. Für die 1960er-Jahre ist ein für die CDU/CSU nachteiliger Bias behauptet worden (Hermens/Unkelbach 1967). Im folgenden Abschnitt wird untersucht, ob es zwischen den Parteien Verzerrungseffekte in der Mehrheitswahlkomponente gibt.

\section{c) Ein parteipolitischer Bias? $\rho$ und $\beta$ im Vergleich zwischen den Parteien}

Wird eine Partei in der Mehrheitskomponente des deutschen Mischwahlsystems bevorzugt bzw. benachteiligt? Für die 1960er-Jahre behaupten etwa Hermens und Unkelbach (1967) eine relative Benachteiligung der Union, ohne ihre 
Behauptung allerdings empirisch zu belegen. Der Bias eines Wahlsystems kann durch den Wahlkreiszuschnitt, die unterschiedliche Wahlkreisgröße (gemessen an der Zahl der Wahlberechtigten) oder durch die regionale Ungleichverteilung von Wählerschaften verursacht werden. Da das Bundeswahlgesetz eine automatische Anpassung der Wahlkreise an veränderte Bevölkerungszahlen vorsieht ( 33 Bundeswahlgesetz, insbesondere Absatz 2, Punkt 2 und 3; Schreiber 1994), und da der Wahlkreiszuschnitt einer unabhängigen, beim Bundeswahlleiter angesiedelten Kommission überantwortet ist, dürften diese beiden Disproportionalitätsfaktoren im bundesdeutschen Wahlsystem keine besonders ausgeprägte Rolle spielen. Einen größeren Einfluss - so steht zu vermuten - haben die regionalen Unterschiede in der jeweiligen Wählerschaft. Hier sind Parteien mit regional konzentrierter Wählerschaft durch die Mehrheitswahl strukturell benachteiligt, denn sie gewinnen ihre Distrikte mit weitem Abstand, verschenken also zu viele Stimmen. Dies sind herkömmlich „linke“ Parteien mit einer Wählerschaft in den dicht besiedelten industriellen Zentren (Rodden 2010; Gudgin/Taylor 1979). Für den bundesdeutschen Fall ließe sich jedoch gleichermaßen vorstellen, dass die CDU/CSU Wahlkreise beispielsweise mit einem hohen Katholikenanteil mit einem „zu weiten“ Abstand gewinnt.

Ein erster Zugang zur Beantwortung der Frage nach einem möglichen parteipolitischen Bias ergibt sich erneut bei der vergleichenden Betrachtung der Erststimmenanteile. Grundlage hierfür ist ein Datensatz mit den Wahlkreisergebnissen der Bundestagswahlen von 1953 bis 2005 (3996 Beobachtungen). ${ }^{8}$ Als erster Befund zeigt sich, dass in diesem Zeitraum die CDU/CSU 2295 und die SPD 1701 dieser fast viertausend Wahlkreise gewonnen hat. Im Durchschnitt errang die Union also pro Wahl 39,6 mehr Direktmandate als die SPD. Aber mit welchem Abstand wurden die Wahlkreise gewonnen? Abbildung 6 zeigt die Dichtefunktionen des Abstands der Erststimmen zwischen SPD und CDU/CSU in den von ihnen gewonnenen Wahlkreisen, Abbildung 6a die Verteilung der Erststimmenanteile für alle Wahlkreise.

8 Es ergeben sich marginale Abweichungen zur exakten Zahl der Wahlkreise in diesen 15 Bundestagswahlen aufgrund vereinzelter Kodierungsfehler für die 2005er- und die 1953er-Bundestagswahl. 


\section{Abbildung 6}

Dichtefunktion des Abstands der Erststimmenanteile zwischen SPD und CDU/ CSU in den von ihnen gewonnenen Wahlkreisen, 1953-2005

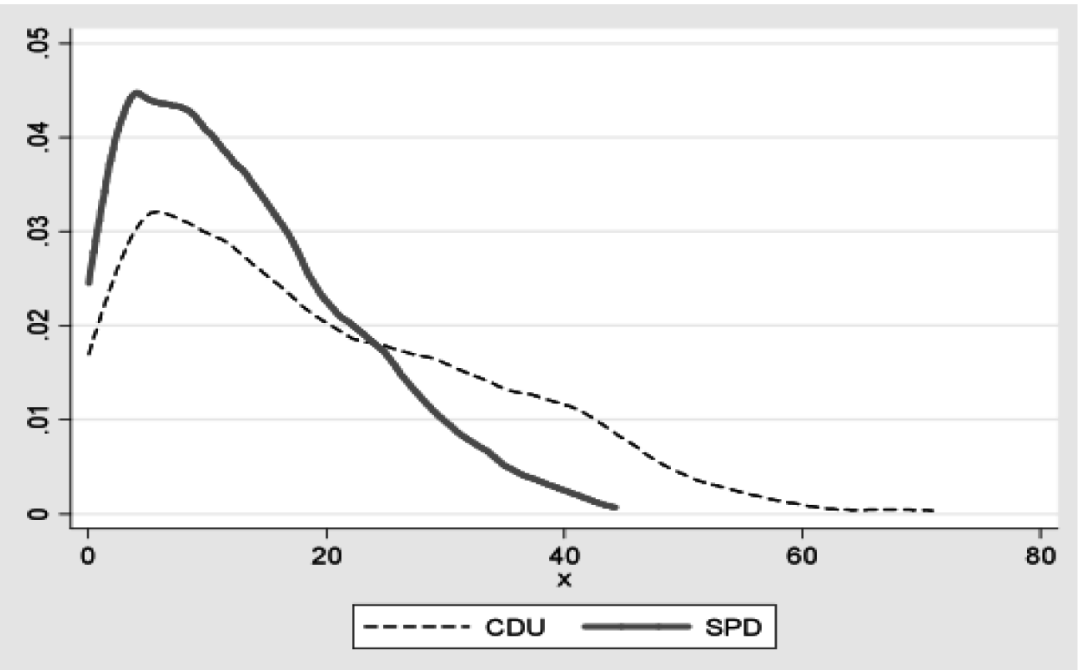

Abbildung 6a

Dichtefunktion der Erststimmenanteile von SPD und CDU/CSU, 1953-2005

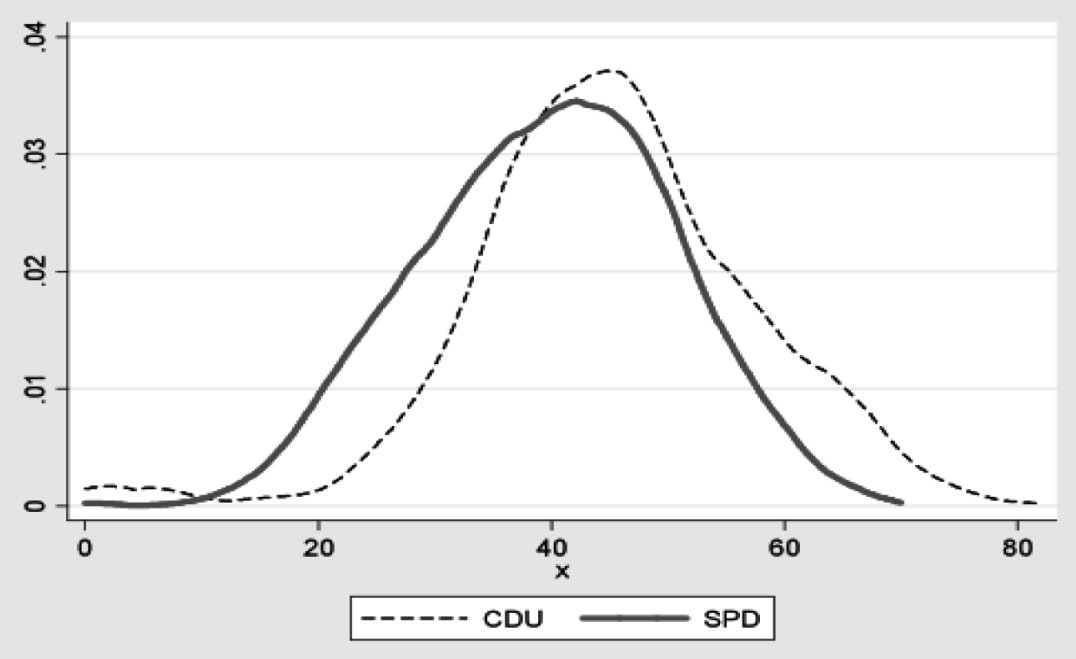


An beiden Schaubildern lässt sich ersehen, dass die SPD ihre Erststimmen offensichtlich „effizienter“ nutzt. Der Anteil der von ihr knapp gewonnenen Wahlkreise ist höher, der Anteil der mit weitem Abstand gewonnenen Wahlkreise dementsprechend geringer als bei der CDU/CSU. Der Vergleich der Erststimmenanteile würde also - wenn überhaupt - eher die CDU/CSU als die SPD in der Mehrheitswahlkomponente des deutschen Wahlsystems benachteiligt sehen. Aber wie übersetzt sich der ungleiche Erfolg in den Wahlkreisen in die Kubusregel-Funktion beider Parteien?

Eine parteipolitische Verzerrung lässt sich ermitteln, wenn wir die erweiterte Cube Rule-Funktion nach Parteien getrennt schätzen und dann die Parameter $\rho$ und $\beta$ vergleichen. Dabei ist eine Partei c. p. dann im Vorteil, wenn die Kubusregel-Funktion stärker nach links verschoben ist (wenn also ihr kritischer Mehrheitswert $\beta$ höher ist), weil sie Direktmandate schon mit einem geringeren Erststimmenanteil erringt. Was die Steigung $\rho$ der Funktion angeht, so ist eine Partei mit einem höheren $\rho$ unterhalb des kritischen Mehrheitswerts im Nachteil (weil sie keine oder kaum Wahlkreise gewinnen kann), oberhalb im Vorteil (weil sie nun nahezu alle Wahlkreise gewinnt). Schätzt man $\rho$ und $\beta$ für SPD und CDU für alle 16 Bundestagswahlen (195 Beobachtungen pro Partei), so ergeben sich für die CDU/CSU Werte von 4,08 $(\rho)$ und 0,076 ( $\beta)$, für die SPD Werte von 4,11 ( $\rho$ ) und 0,094 ( $\beta$ ). Der Steigungsparameter ist also fast identisch, hingegen ist die Kubusregel-Funktion der SPD etwas weiter nach links gerückt. In Abbildung 7 ist entsprechend der leichte elektorale Vorteil der SPD gegenüber der CDU erkennbar: Bei gleichem Erststimmenanteil liegt der Anteil an gewonnenen Direktmandaten der SPD über den gesamten Kurvenverlauf leicht über dem der CDU. Eine wahlregelbedingte Benachteiligung der SPD bei der Übersetzung von Erststimmen in Direktmandate bestätigt sich mithin empirisch nicht, auch wenn es der SPD grundsätzlich schwerer zu fallen scheint, solche Mandate zu erringen. Wenn überhaupt, legen die Daten eine leichte Benachteiligung der CDU nahe.

Die Längsschnittbetrachtung ergibt kein grundsätzlich anderes Bild. Tabelle 1 bietet einen Überblick für alle Bundestagswahlen. Das zunehmende Auftreten sehr polarisierter Wahlen, bei denen eine Partei in einem Land (fast) alle, die andere Partei (fast) keine Direktmandate erringt (s. u.), verhindert die Berechnung der parteispezifischen $\rho$ und $\beta$-Parameter für die letzten Bundestagswahlen, sodass ab 1998 kein direkter Vergleich zwischen SPD und CDU/CSU mehr möglich ist. Für die 1960er-Jahre bestätigt sich jedoch der leichte elektorale Nachteil der Union, den Hermens und Unkelbach 1967 behauptet hatten (Tabelle 1). 
Tabelle 1

Parteipolitischer Bias in der Mehrheitskomponente des deutschen Mischwahlsystems, $\rho$ und $\beta$ für CDU/CSU und SPD in den Bundestagswahlen von 1953 bis 2009

\begin{tabular}{cclll}
\hline Bundestagswahl & SPD $\boldsymbol{\rho}$ & SPD $\boldsymbol{\beta}$ & CDU $\boldsymbol{\rho}$ & \multicolumn{1}{c}{ CDU $\boldsymbol{\beta}$} \\
\hline 1953 & 4.474566 & 0.1224502 & 4.1593250 & 0.13380919 \\
1957 & 6.901213 & 0.1133053 & 3.901467 & 0.1094263 \\
1961 & 9.726095 & 0.1110655 & 6.516785 & 0.0906508 \\
1965 & 8.402094 & 0.0710321 & 7.016078 & 0.0438249 \\
1969 & 5.488655 & 0.0621457 & 6.264926 & 0.0365849 \\
1972 & 4.783949 & 0.041750 & 4.855561 & 0.0165645 \\
1976 & 6.578847 & 0.0500192 & 6.359261 & 0.0243045 \\
1980 & 6.782722 & 0.0473623 & 8.551348 & 0.0507163 \\
1983 & 10.58924 & 0.0380498 & 8.869953 & 0.0325486 \\
1987 & 8.197452 & 0.0628768 & 8.065799 & 0.0598539 \\
1990 & 6.009919 & 0.0895037 & 6.272394 & 0.0978378 \\
1994 & 7.199286 & 0.0791624 & 5.895126 & 0.0960033 \\
1998 & $* * *$ & $* * *$ & 5.033498 & 0.0499634 \\
2002 & 6.222764 & 0.1204042 & $* * *$ & $* * *$ \\
2005 & 3.110234 & 0.1640065 & $* * *$ & $* * *$ \\
2009 & 9.295541 & 0.155284 & $* * *$ & $* * *$
\end{tabular}

Betrachten wir abschließend die zwei Funktionsparameter $\rho$ und $\beta$ gemeinsam, so lässt sich festhalten, dass die hohe Zahl der Wahlkreisparteien unzweifelhaft den stärksten Einfluss auf die Disproportionalität in der Mehrheitskomponente des deutschen Mischwahlsystems ausübt - und dieser Faktor wirkt insbesondere auf die Linksverschiebung der Kubusregel-Funktion, nicht auf ihre Steigung. Im folgenden Abschnitt gehe ich näher auf die Folgen der Disproportionalität ein - etwa hinsichtlich der internen Zusammensetzung der Parlamentsfraktionen oder der Entstehung von Überhangmandaten. 
Abbildung 7

Die Kubusregel-Funktion für SPD und CDU/CSU, alle Bundestagswahlen

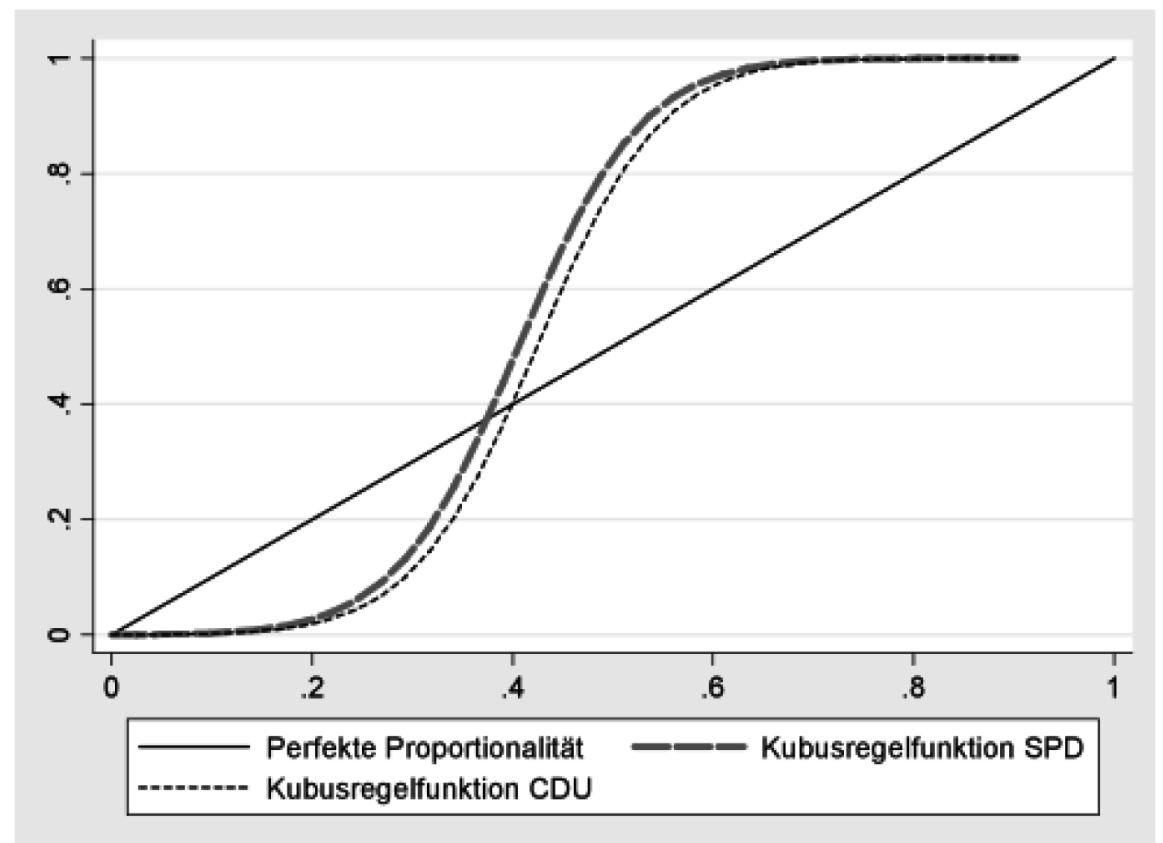

\section{Folgen der Disproportionalität}

Eine wichtige Folge einer disproportionalen Übersetzung von Stimmen in Sitze, die in der Literatur allerdings bislang kaum Erwähnung gefunden hat, betrifft die Fraktionszusammensetzung nach Direkt- und Listenkandidaten für diejenigen Parteien, für die Direktmandate überhaupt eine Rolle spielen - für die SPD und die CDU/CSU. Hinter diesem Befund steht folgender Zusammenhang: Gewinnt eine der beiden großen Parteien (relativ zur anderen) hinzu, so übersetzt sich dieser Stimmengewinn in einen stärkeren Zugewinn an Direktmandaten. Da die große Partei mit Stimmenzugewinn eine erhöhte Wahrscheinlichkeit der Regierungsbeteiligung hat als die mit Stimmenverlusten, ergibt sich das Muster, dass sich die größte Regierungsfraktion zu etwa 3/5 oder 2/3 aus Wahlkreisabgeordneten zusammensetzt, während für die große Oppositionspartei genau umgekehrt gilt, dass sie weit überwiegend mit Listen- 
abgeordneten im Parlament vertreten ist (Manow/Nistor 2009). Abbildung 8 zeigt diese Entwicklung. In Zeiten mit CDU-Regierungsbeteiligung sind im Durchschnitt 73,1 aller CDU/CSU Abgeordneten direkt gewählt, in Zeiten der christdemokratischen Opposition nur etwa 49,6 \%. Stellt die SPD die stärkste Regierungsfraktion, so sind etwas sechzig Prozent (62,3\%) ihrer Abgeordneten direkt im Wahlkreis gewählt, in Oppositionszeiten nur etwa 39,8 \% (die Zeiten der Großen Koalition 2005 bis 2009 sind für beide Parteien als Regierungsbeteiligung nicht einberechnet).

\section{Abbildung 8}

Verhältnis von Direktabgeordneten zu allen Fraktionsabgeordneten von CDU/ CSU und SPD, 2. bis 17. Wahlperiode

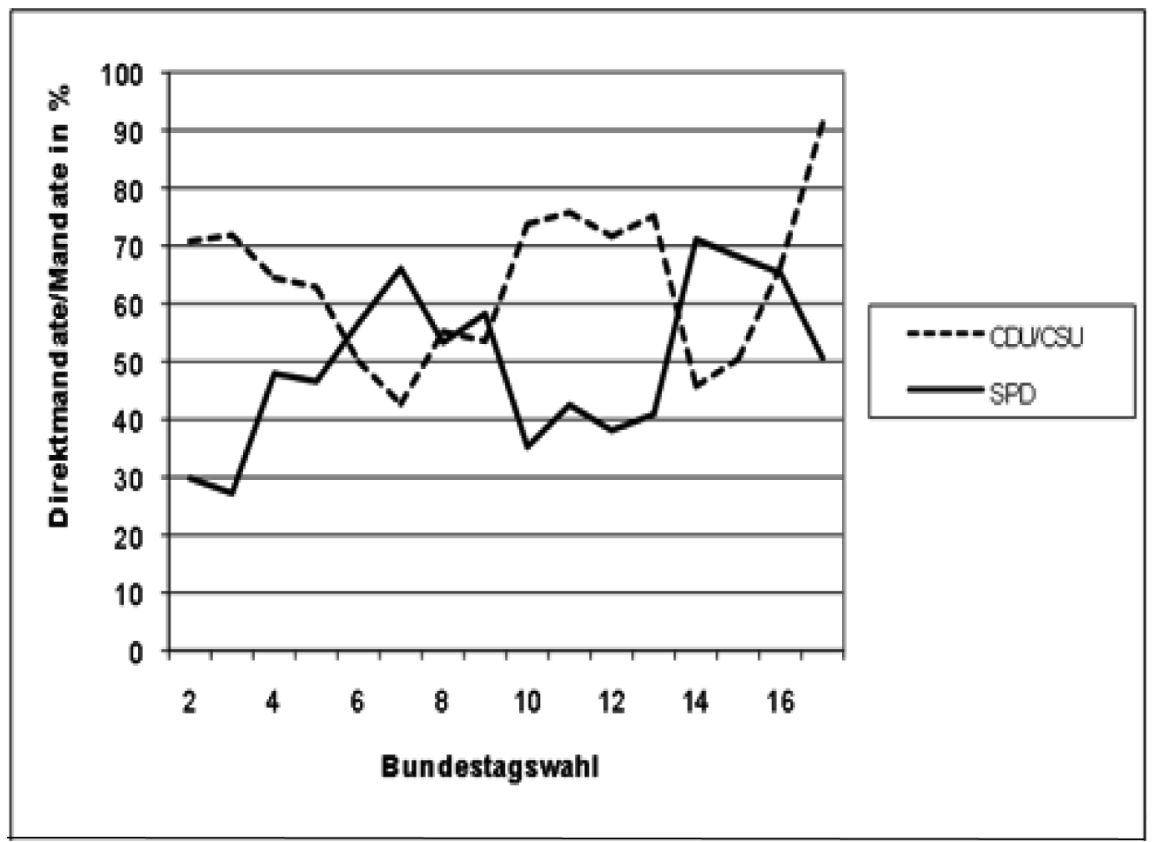

Eine weitere Entwicklung betrifft die Polarisierung der Direktmandateverteilungen. Dort, wo eine Partei alle und die andere keinen Wahlkreis direkt gewinnen kann, wird es wahrscheinlicher, dass der Anteil an Direktmandaten mehr als doppelt so hoch ist wie der Zweitstimmenanteil - eine Voraussetzung 
für das Auftreten von Überhangmandaten (Behnke 2007). Wie die Box PlotGrafik der Abbildung 9 zeigt, war diese Polarisierung tatsächlich in den ersten Bundestagswahlen und nun erneut in den letzten besonders ausgeprägt. ${ }^{9}$

\section{Abbildung 9}

Polarität der Erststimmenanteile von CDU/CSU und SPD, 16 Bundestagswahlen (1953-2009), 9 bis 16 Bundesländer

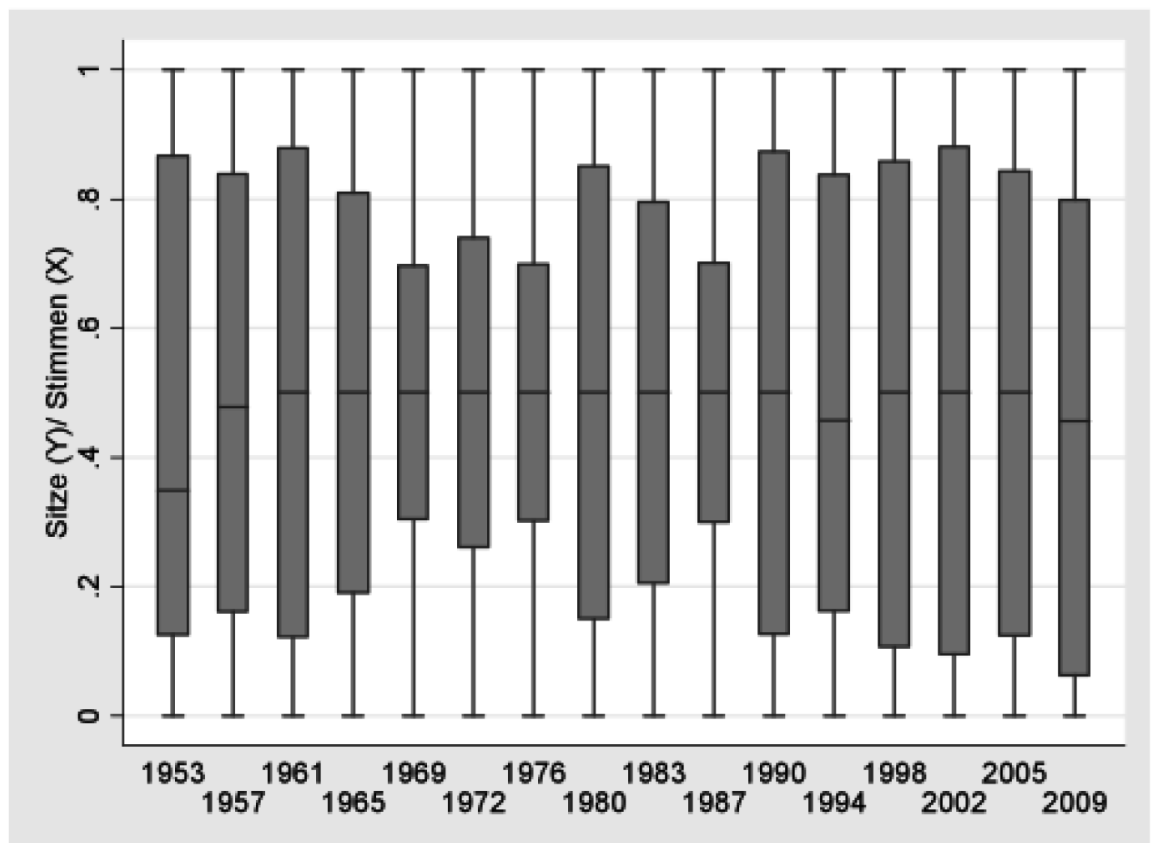

Eine Folge der Zunahme polarisierter Direktmandateverteilungen betrifft die Nominierungsstrategien der Parteien: Sie müssen nicht nur ihre Direktkandidaten vor dem Eventualfall eines schlechten Wahlergebnisses schützen (durch eine zusätzliche Absicherung auf guten Listenplätzen), sondern sie müssen

9 In diesem Zusammenhang zeigt sich auch, dass Überhangmandate wie eine Prämie für den Wahlgewinner wirken. Sie verstärken einen generellen Wahltrend, belohnen also den Sieger und bieten damit einen durchaus willkommenen Konzentrationseffekt. Eine solche Konzentrationswirkung des Wahlrechts wird in den Diskussionen über die Reform des deutschen Wahlrechts immer wieder als wünschenswerte Wahlrechtseigenschaft genannt (Linhart 2009: 650, Fn. 47). 
auch für den Fall des Wahlerfolgs vorsorgen, wenn die überproportional erfolgreichen Direktkandidaten die Listenplätze der eigenen Partei gefährden. Wie wir gesehen haben, ist diese Gefahr im Verlauf der Zeit gestiegen - Überhangmandate sind ein Indikator genau dieses Phänomens. Die Parteien müssen deshalb im Fall eines Wahlerfolgs auch Kandidaten auf den vorderen Listenplätzen gegenüber den eigenen Direktkandidaten schützen - am besten durch eine Doppelkandidatur, also mit einer zusätzlichen Direktkandidatur in einem aussichtsreichen Wahlkreis. Das Absichern muss also in beide Richtungen erfolgen. Erwarten lässt sich mithin ein Trend zu zunehmenden Doppelkandidaturen - eine Erwartung, die sich empirisch bestätigt (Manow/Nistor 2009).

Abschließend geht es mir um den Zusammenhang zwischen der im Zeitverlauf zunehmenden Disproportionalität in der Mehrheitswahlkomponente des deutschen Wahlsystems und dem Auftreten von Überhangmandaten. Unter den verschiedenen möglichen Verursachungsfaktoren von Überhangmandaten (Grotz 2000; Behnke 2003a, 2009) sollen dabei wiederum die effektive Zahl der Wahlkreisparteien und damit der $\beta$-Parameter im Mittelpunkt des Interesses stehen. Der Effekt von $\beta$ ist in Abbildung 10 gut zu ersehen. In der Abbildung sind diejenigen Bundesländer, in denen Überhangmandate entstanden, mit $\ddot{U}$ gekennzeichnet. Wie ersichtlich, kam es in der 2005er-Bundestagswahl zu Überhangmandaten insbesondere dort, wo Parteien mit Erststimmenanteilen von um die $40 \%$ mehr als $80 \%$ aller Direktmandate errungen haben. Da der Erststimmenanteil der beiden großen Parteien, die als einzige regelmäßig Direktmandate gewinnen - die Union und die SPD - wegen des Stimmensplittings grundsätzlich höher ausfällt als ihr Zweitstimmenanteil, fällt die für die Entstehung von Überhangmandaten relevante Diskrepanz zwischen dem Direktmandateanteil und dem Zweitstimmenanteil noch größer aus als die gezeigte Diskrepanz zwischen Erststimmen und Direktmandaten. Abbildung 10 bestätigt die Faustregel: „Überhangmandate entstehen dann, wenn der Anteil der durch eine Partei errungenen Direktmandate an allen Direktmandaten mehr als doppelt so hoch ausfällt wie der Anteil der Zweitstimmen dieser Partei in diesem Bundesland“" (Behnke 2007: 187).

Überhangmandate sind insbesondere Folge der „Entscheidungsregeldivergenz" (Grotz) im deutschen Mischwahlsystem, also der höheren Disproportionalität der Mehrheitswahl im Vergleich zur Verhältniswahl. Die Entscheidungsregeldivergenz allein macht ihr Auftreten jedoch noch nicht wahrscheinlich, hierzu müssen weitere Bedingungen erfüllt sein: „,[B]ei einem Zweiparteiensystem ist das Auftreten von Überhangmandaten auf Grund der Entscheidungsregeldivergenz kaum zu erwarten, da die schwächere von beiden [Parteien] in Folge wahlgeographischer Stimmendifferenzen in der Regel einige Direktman- 
date gewinnt und damit der anderen Partei die Möglichkeit nimmt, einen Überhang zu erzielen. Realistischer wird das [...] allerdings unter einem Drei- bzw. Mehrparteiensystem: Hierbei kann beispielsweise eine drittplatzierte Partei C, die relativ viele Erststimmen in einem Bundesland auf sich vereinigt hat, der stärksten Partei A gleichsam dazu verhelfen, viele Wahlkreismandate mit relativ geringen Stimmenmehrheiten gegenüber der zweitplatzierten Partei B zu gewinnen. Damit wird ein Überhang für Partei A [...] durchaus wahrscheinlich“ (Grotz 2000: 713)

\section{Abbildung 10}

Erststimmenanteile und Anteile an den Direktmandaten für Union und SPD in der 2005er-Bundestagswahl ( $\ddot{U}=$ Länder mit Überhangmandaten)

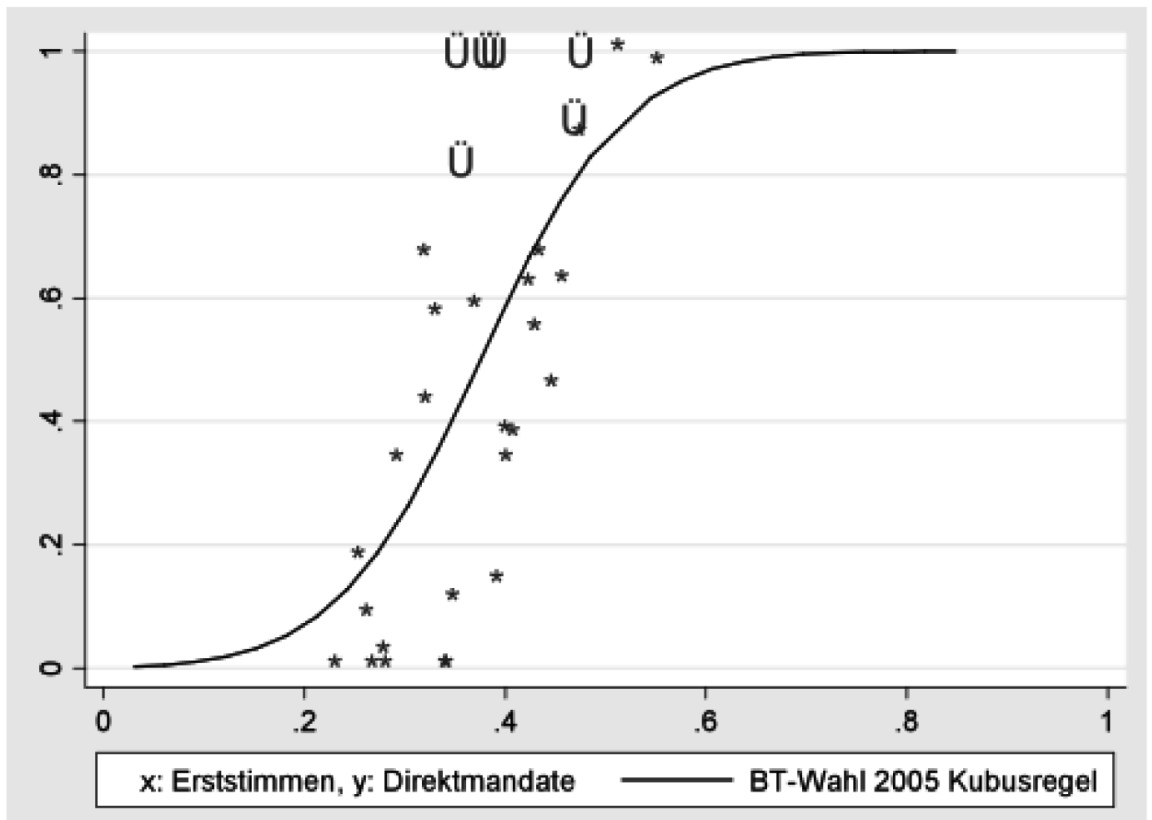

Das Ausmaß der Linksverschiebung der Kubusregel-Funktion, die die effektive Zahl der Wahlkreisparteien anzeigt, sollte daher ein guter Erklärungsfaktor für das Auftreten von Überhangmandaten sein, denn umgekehrt gilt auch: Hat eine Partei über $50 \%$ der Zweitstimmen erhalten, kann der Anteil der direkt eroberten Wahlkreise nicht zu Überhangmandaten führen (Behnke 2003a; 
Grotz 2000: 713). In diesem Zusammenhang zeigt sich damit wiederum der Vorteil der Messung der Disproportionalität in der Mehrheitswahlkomponente des deutschen Wahlsystems über die Parameter der Kubusregel-Funktion, denn mit ihnen lässt sich die Divergenz zwischen den Entscheidungsregeln, die Grotz für „schwieriger zu operationalisieren“ hält (2000: 719), recht genau ermitteln: Für die Verhältniswahl gilt ja $\rho=1$ und $\beta \rightarrow 0$ (wegen der 5-Prozenthürde ist $\beta$ etwas größer Null).

Tabelle 2

$\beta$ als Erklärungsfaktor für Überhangmandate (negativ Binomial-Regression)

\begin{tabular}{|c|c|c|}
\hline & (1) & (2) \\
\hline$\beta$-Bundesland & $\begin{array}{l}18.389 \\
(0.000)^{* * *}\end{array}$ & $\begin{array}{l}16.804 \\
(0.010)^{* * *}\end{array}$ \\
\hline$\beta$-Bundestagswahl & $\begin{array}{l}23.082 \\
(0.000)^{* * *}\end{array}$ & $\begin{array}{l}16.905 \\
(0.028)^{* *}\end{array}$ \\
\hline Wahlkreisgröße & & $\begin{array}{l}-0.000 \\
(0.001)^{* * *}\end{array}$ \\
\hline Wahlbeteiligung & & $\begin{array}{c}-10.494 \\
(0.063)^{*}\end{array}$ \\
\hline Stimmensplitting & & $\begin{array}{c}0.000 \\
(0.067)^{*}\end{array}$ \\
\hline Konstante & $\begin{array}{l}-5.833 \\
(0.000)^{* * *}\end{array}$ & $\begin{array}{c}8.554 \\
(0.183)\end{array}$ \\
\hline Beobachtungen & 390 & 390 \\
\hline
\end{tabular}

p-Werte in Klammern; * signifikant auf dem 10\%-Niveau; ** signifikant auf dem 5\%-Niveau; $* * *$ signifikant auf dem 1\%-Niveau. Pseudo-R ${ }^{2}:=0,12$; Prob $>\chi^{2}=0,000$.

Es wäre also nicht überraschend, wenn der $\beta$-Parameter der modifizierten Kubusregel-Funktion sich als ein guter Prädiktor für das Auftreten von Überhangmandaten erweist. Inwiefern bestätigt sich diese Erwartung? Methodisch ist bei der Antwort auf diese Frage zu beachten, dass die abhängige Variable (Zahl der Überhangmandate) eine diskrete, nicht-negative (Zähl-)Variable ist, deren Verteilung durch Überdispersion gekennzeichnet ist (mit einem Standardfehler, der größer als der Mittelwert ist). Daher kann der Einfluss von $\beta$ auf die Wahrscheinlichkeit des Auftretens von Überhangmandaten nicht mit einer 
OLS-Regression ermittelt werden. Ich verwende stattdessen eine negative Binomial-Regression (siehe Tabelle 2).$^{10}$ Ich untersuche zunächst nur den Einfluss von $\beta$ und kontrolliere in einer zweiten Modellspezifikation für andere mögliche Determinanten des Auftretens von Überhangmandaten, die in der Literatur genannt werden: für das Ausmaß des Stimmensplittings, für Unterschiede in der Wahlbeteiligung und für die Wahlkreisgröße - gemessen als durchschnittliche Zahl der Wahlberechtigten pro Wahlkreis (Behnke 2003a). Das Ausmaß des Stimmensplittings wird hier als Summe der absoluten Werte aus den Differenzen der Erst- und Zweitstimmen für die CDU/CSU, SPD, FDP, Grüne und PDS/Die Linke, geteilt durch die Zahl der Parteien, operationalisiert. Die Wahlkreisgröße bemisst sich nach der Zahl der Wahlberechtigten, die Wahlbeteiligung nach der Zahl der ungültigen und gültigen Stimmen als Anteil der Zahl der Wahlberechtigten. Hierbei sind immer die Durchschnittswerte auf Landesebene ermittelt worden.

Die Regression bestätigt den starken und stabilen Einfluss des $\beta$-Parameters auf die Wahrscheinlichkeit des Auftretens von Überhangmandaten. Auch bei Einschluss anderer Erklärungsfaktoren behalten die beiden Variablen ihre Signifikanz - wenn auch die Koeffizientenstärke (leicht) abnimmt. Zugleich bestätigen sich alle in der Literatur getroffenen Vermutungen über die weiteren Determinanten für das Auftreten von Überhangmandaten (vgl. etwa Grotz 2000): Eine geringe Wahlbeteiligung erhöht die Wahrscheinlichkeit von Überhangmandaten, erhöhtes Stimmensplitting steigert die Wahrscheinlichkeit ihres Auftretens. Je kleiner der Wahlkreis gemessen an der Zahl der Wahlberechtigten, desto höher die Wahrscheinlichkeit von Überhangmandaten.

\section{Zusammenfassung}

Wie werden in der Mehrheitswahlkomponente des bundesdeutschen Mischwahlsystems Stimmen in Sitze übersetzt? Zunächst (Abbildung 1) zeigte sich auch im deutschen Wahlsystem hinsichtlich der Erststimmen und Direktmandate das disproportionale Muster der bekannten Kubusregel. Doch die nähere Betrachtung konnte die erheblichen Abweichungen von diesem Muster auf der Ebene der sogenannten Unterverteilung verdeutlichen, also auf der Ebene der Bundesländer, auf der im deutschen Wahlsystem der Abgleich zwischen Erstund Zweitstimmen vorgenommen wird. Diese Abweichungen sind insbesondere in zweierlei Hinsicht ausgeprägt: erstens hinsichtlich der Steigung der Kubusregel-Funktion, die auf Landesebene steiler ausfällt als auf nationaler Ebene

10 Eine zero-inflated negative Binomial kommt im Wesentlichen zu gleichen Ergebnissen. 
und mit der „Distriktgröße“, d. h. mit der Zahl von Wahlkreisen pro Land, variiert; zweitens hinsichtlich der zunehmenden Linksverschiebung der Kurve, die insbesondere seit der Deutschen Einigung die Entwicklung des deutschen Parteiensystems von einem 3- zu einem 5-Parteiensystem widerspiegelt. Da alle Parteien, die im Bundestag vertreten sind, im Regelfall auch in jedem Wahlkreis mit eigenen Kandidaten antreten, übersetzt sich diese Zunahme der Parteienzahl auch in eine höhere Zahl an Wahlkreisparteien, also derjenigen Parteien, die sich um ein Direktmandat bewerben. Dies aber erhöht die Disproportionalität der Mehrheitswahlkomponente: Eine hohe Zahl an Wahlkreisen kann nun auch schon mit Erststimmenanteilen von nur knapp über $30 \%$ gewonnen werden. Die Vergleiche zwischen Vor- und Nachvereinigungswahlen (Abbildung 2), zwischen Bundesländern mit viel und wenig Wahlkreisen (Abbildung 5), und zwischen den Bundestagswahlergebnissen in den ostdeutschen und westdeutschen Bundesländern nach 1990 (Abbildung 4) verdeutlichten diesen Effekt. Der Vergleich der Kubusregel-Funktion zwischen SPD und CDU/CSU ergab jedoch nur sehr geringe Hinweise auf eine wahlregelspezifische Verzerrung im Prozess der Übersetzung von Stimmen in Sitze - und dieser Bias wirkt entgegen den Erwartungen (Rodden 2010) eher zulasten der Union (Abbildung 7).

Im zweiten Teil des Aufsatzes ging es um die Folgen der Disproportionalität. Hierbei standen drei Auswirkungen der Disproportionalität im Vordergrund: 1) Auswirkungen auf die interne Zusammensetzung der beiden großen Parlamentsfraktionen von SPD und CDU/CSU nach Listen- und Direktabgeordneten je nach Regierungsstatus der Fraktion; 2) auf die Nominierungsstrategien der Parteien, die regelmäßig Direktmandate gewinnen, nämlich von SPD und CDU/CSU. Wie gezeigt, müssen die Parteien mit ihren Nominierungsstrategien darauf reagieren, dass nicht nur Direktkandidaten mit guten Listenplätzen abgesichert werden müssen (im Fall der Niederlage), sondern auch Listenkandidaten vor dem Erfolg der eigenen Direktkandidaten geschützt werden müssen (im Falle des Wahlsiegs). Schließlich ging es 3) um die Untersuchung der Determinanten von Überhangmandaten. Wie gezeigt, hat die höhere effektive Zahl der Wahlkreisparteien, die sich in einem erhöhten $\beta$ der Kubusregel-Funktion widerspiegelt, einen sehr starken und sehr robusten Einfluss auf die Eintrittswahrscheinlichkeit des Ereignisses „Überhangmandat“. Dieser Einfluss bleibt auch signifikant, wenn andere Erklärungsfaktoren in die Betrachtung einbezogen werden (Tabelle 2, oben).

Ich möchte abschließend auf zwei Implikationen des Arguments zu sprechen kommen. Zunächst zeigt sich eine seit 1990 zunehmende Abweichung 
von den Effekten einer reinen Verhältniswahl durch die zuletzt sehr hohe Zahl an Überhangmandaten. Aber auch unabhängig von der numerischen parteipolitischen Zusammensetzung im Parlament zeigen sich Effekte im Zusammenwirken der beiden Wahlrechtskomponenten, die für die Kandidatenstrategien, für die parteipolitische Nominierungspraxis und schließlich auch für die Wähler bedeutsam sind. Die zunehmenden Abweichungen von der rein numerischen Proportionalität - durch die Überhangmandate - sind selbst natürlich auch maßgeblich durch das Zusammenwirken beider Wahlsystemkomponenten im deutschen Wahlsystem verursacht. Alles dies spricht dafür, seiner Mehrheitswahlkomponente stärkere Aufmerksamkeit zu widmen. Ihr Effekt erschöpft sich ganz offensichtlich nicht in der schlichten Personalisierung einer sich ansonsten strikt nach Verhältniswahlprinzipien vollziehenden Übersetzung von Stimmen in Sitze.

Des Weiteren hat das dargelegte Argument auch Implikationen für unser Verständnis der dominierenden Repräsentationslogik im deutschen Wahlsystem. In der Kontroverse über die angebliche Rollendifferenzierung zwischen Listenund Direktabgeordneten ${ }^{11}$ bietet die hier berichtete Empirie Unterstützung für jene Position, die eine ausgeprägte Rollendifferenzierung zwischen beiden Abgeordnetentypen für eher unwahrscheinlich hält. Angesichts der hohen Bedeutung der Doppelkandidaturen und der höheren Gefährdung der Direktkandidaten bei geringen Stimmschwankungen müssen Abgeordnete sowohl die Interessen des Wahlkreises als auch die Programmatik der Partei im Auge behalten, wollen sie ihre Wiederwahlchancen maximieren.

\section{Literatur}

Behnke, Joachim, 2003a: Ein integrales Modell der Ursachen von Überhangmandaten, in: PVS 44, 41-65.

Behnke, Joachim, 2003b: Überhangmandate: Ein behebbarer Makel im institutionellen Design des Wahlsystems, in: ZPol 13 (3),1235-1269.

Behnke, Joachim, 2003c: Von Überhangsmandaten und Gesetzeslücken, in: APuZ, B 52, 21-28.

Behnke, Joachim, 2007: Das Wahlsystem der Bundesrepublik Deutschland. Logik, Technik und Praxis der Verhältniswahl, Baden-Baden.

11 U. a. Stratmann/Baur 2002; Lancaster/Patterson 1990; Patzelt 2007; Klingemann/ Wessels 2001. 
Behnke, Joachim, 2009: Überhangmandate bei der Bundestagswahl 2009. Eine Schätzung mit Simulationen, in: ZParl 40, 620-636.

Bücking, Hans-Jörg, 1998: Der Streit um Grundmandatsklausel und Überhangmandate, in: Eckhard Jesse/Konrad Löw (Hrsg.), Wahlen in Deutschland, Berlin.

Cox, Gary W., 1997: Making Votes Count. Strategic Coordination in the World's Electoral Systems, New York.

Cox, Karen E./Schoppa, Leonard J., 2002: Interaction Effects in Mixed-Member Electoral Systems: Theory and Evidence from Germany, Japan, and Italy, in: Comparative Political Studies 35 (9), 1027-1053.

Fürnberg, Ossip/Knothe, Danko, 2009: Wahlsiege ohne Stimmenmehrheit: Auswirkungen von verstärktem „Lagersplitting“ auf Mandatsverteilung und Koalitionsoptionen, in: ZParl 40, 56-74.

Grotz, Florian, 2000: Die personalisierte Verhältniswahl unter den Bedingungen des gesamtdeutschen Parteiensystems. Eine Analyse der Entstehungsursachen von Überhangmandaten seit der Wiedervereinigung, in: PVS 41, 707-729.

Gudgin, Graham/Taylor, Peter J., 1979: Seats, Votes, and the Spatial Organization of Elections, London.

Hainmüller, Jens/Kern, Holger Lutz, 2008: Incumbency as a Source of Spillover Effects in Mixed Electoral Systems: Evidence from a Regression Discontinuity Design, in: Electoral Studies 27, 213-227.

Hainmüller, Jens/Kern, Holger Lutz/Bechtel, Michael, 2006: Wahlkreisarbeit zahlt sich doppelt aus - Zur Wirkung des Amtsinhaberstatus einer Partei auf ihren Zweitstimmenanteil bei den Bundestagswahlen 1949 bis 1998, in: Thomas Bräuninger/Joachim Behnke (Hrsg.), Jahrbuch für Handlungsund Entscheidungstheorie. Band 4: Schwerpunkt Parteienwettbewerb und Wahlen, Wiesbaden.

Hermens, Ferdinand A./Unkelbach, Helmut, 1967: Die Wissenschaft und das Wahlrecht, in: PVS 8, 2-22.

Jesse, Eckhard, 1990: Elections. The Federal Republic of Germany in Comparison, New York/Oxford/Munich.

Jesse, Eckhard, 1998: Grundmandatsklausel und Überhangmandate. Zwei wahlrechtliche Eigentümlichkeiten in der Kritik, in: Max Kaase/Hans-Dieter Klingemann (Hrsg.), Wahlen und Wähler. Analysen aus Anlass der Bundestagswahl 1994, Opladen.

Kendall, M. G./Stuart, A., 1950: The Law of Cubic Proportions in Electoral Results, in: British Journal of Sociology 1 (3), 183-197. 
Kendall, M. G./Stuart, A., 1952: La Loi de Cube dans les Elections Britanniques, in: Revue Francaise de Science Politique 2, 270-276.

King, Gary, 1990: Electoral responsiveness and partisan bias in multiparty democracies, in: Legislative Studies Quarterly 15 (2), 159-181.

King, Gary/Browning, Robert X., 1987: Democratic Representation and Partisan Bias in Congressional Elections, in: American Political Science Review 81 (4), 1251-1273.

Klingemann, Hans-Dieter/Wessels, Wolfgang, 2001: The Consequences of Germany's Mixed-Member System: Personalization at the Grassroots?, in: Matthew S. Shugart/Martin P. Wattenberg (Hrsg.), Mixed-Member Electoral Systems. The Best of two Worlds?, Oxford.

Laakso, Markku, 1979; Should a Two-and-a-Half Law Replace the Cube Law in British Elections?, in: British Journal of Political Science 9, 355-384.

Lancaster, Thomas D./Patterson, William D, 1990: Comparative Pork Barrel Politics Perceptions from the West-German-Bundestag, in: Comparative Political Studies 22 (4), 458-477.

Linhart, Eric, 2009: Mögliche Auswirkungen von Grabenwahlsystemen in der Bundesrepublik Deutschland. Theoretische Überlegungen und Simulationen, in: ZParl 40, 637-660.

Mager, Ute/Uerpmann, Robert, 1995: Überhangmandate und Gleichheit der Wahl, in: Deutsches Verwaltungsblatt 110, 273-280.

Mann, Gerald H., 1995: Die unumgängliche Umkehr bei der Berechnung von Überhangmandaten: Reformvorschläge, in: ZParl 27, 398-404.

Manow, Philip, 2010a: Dimensionen der Disproportionalität. Erststimmen und Direktmandate in den Bundestagswahlen von 1953 bis 2009, in: PVS 60 (3), i. E.

Manow, Philip, 2010b: Quasi-Incumbency effects, or: Why do small parties enter hopeless district races? Evidence from Germany's mixed-electoral sytem (unter Begutachtung).

Manow, Philip/Martina Nistor, 2009: Wann ist ein Listenplatz sicher? Eine Untersuchung der Bundestagswahlen 1953 bis 2002, in: ZParl 40, 603-620.

Naundorf, Christian, 1996: Der überflüssige Überhang: Reformvorschläge, in: ZParl 27, 393-397.

Patzelt, Werner, 2007: The Constituency Roles of MPs at the Federal and Länder Levels in Germany, in: Regional and Federal Studies 17 (1), 47-70.

Rodden, Jonathan, 2010: The Geographic Distribution of Political Preferences, in: Annual Review of Political Science 13, 321-340. 
Schreiber, Wolfgang, 1994: Handbuch des Wahlrechts zum Deutschen Bundestag. Kommentar zum Bundeswahlgesetz, Köln/Berlin/Bonn/München (5. völlig überarb. Auflage).

Stratmann, Thomas/Baur, Martin, 2002: Plurality Rule, Proportional Representation, and the German Bundestag: How Incentives to Pork-Barrel Differ Across Electoral Systems, in: American Journal of Political Science 46 (3), 506-514.

Taagepera, Rein/Shugart, Matthew S., 1989: Seats/Votes. The Effects and Determinants of Electoral Systems, New Haven.

Korrespondenzanschrift:

Prof. Dr. Philip Manow

Moderne Politische Theorie

Institut für Politische Wissenschaft

Ruprecht Karls-Universität Heidelberg

Bergheimer Str. 58

69115 Heidelberg

E-Mail: pmanow@urz.uni-heidelberg.de

Web: http://www.uni-heidelberg.de/politikwissenschaften/personal/manow/

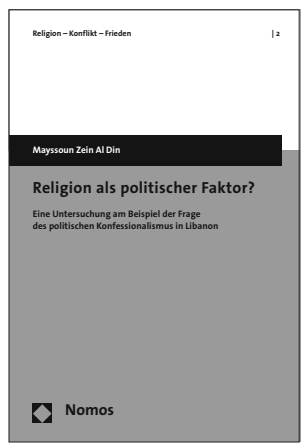

\section{Religion als politischer Faktor?}

Eine Untersuchung am Beispiel der Frage des politischen Konfessionalismus in Libanon

Von Mayssoun Zein Al Din

2010, 290 S., brosch., 44,- $€$,

ISBN 978-3-8329-5360-7

(Religion - Konflikt - Frieden, Bd. 2)

Bitte bestellen Sie im Buchhandel oder

versandkostenfrei unter $\downarrow$ www.nomos-shop.de

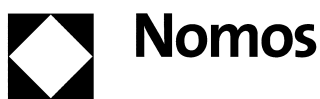

